\title{
Natural history of liver fibrosis progression in patients confected with hepatitis $c$ virus and human immunodeficiency virus type 1
}

\author{
Pranab Kumar Bhattacharya ${ }^{[1]}$ and Sumana Mukherjee ${ }^{[2]}$
}

[1]. Department of Pathology, Murshidabad District Medical College, Beharampore, Murshidabad, West Bengal, India. [2]. Department of Pathology, Calcutta School of Tropical Medicine Kolkata, West Bengal, India.

\section{The Editor,}

We reviewed the major article by Pirellis $^{(\mathbf{1})}$ et al regarding hepatic fibrosis progression in the presence of hepatitis $\mathrm{C}$ virus (HCV) infection in patients with human immunodeficiency virus-1 (HIV-1), which reported greater stimulation by hepatic stellate cells on liver fibrosis progression in patients co infected with HIV-1 and HCV, owing to increased expression of transforming growth factor beta (TGF)- $\beta$ and no relation with human platelet antigen polymorphism ${ }^{(\mathbf{1})}$.

Hepatitis $\mathrm{C}$ viral infection is a common co infection in HIV1 -infected individuals, as is hepatitis B virus (HBV), Torque teno virus (TTV), and SEN virus. Co infected patients tend to have high hepatitis $\mathrm{C}$ virus-ribonucleic acid (HCV RNA) levels and more severe liver disease, faster disease progression, and a higher prevalence of hepatic fibrosis ${ }^{(2)}$. Quantitative assessment of HCV RNA should be performed using reverse transcription polymerase chain reaction, which is easier and more commercially available than transcription mediated assays (TAM); however, TAM is an extremely sensitive technique.

Factors that determine $\mathrm{HCV}$ viral clearance or persistence in the liver remain unclear. Of acute $\mathrm{HCV}$ infections, $80 \%$ become chronic, with elevated alanine aminotransferase (ALT) levels, and most patients do not show physical signs of liver disease. Elevated aminotransferase levels, particularly that of ALT, are commonly observed, although one third of asymptomatic patients may have a persistently normal ALT level. Only 5\% of patients with chronic HCV infection have symptomatic liver disease, which usually includes mild and insidious fatigue and dull intermittent pain in the upper quadrant, while anorexia, nausea, pruritus, arthralgia, and myalgia are less common. The disease progresses slowly, if at all, in most patients; $20 \%$ of patients progress to cirrhosis in 20 years, and progression can

Corresponding author: Prof. Pranab Kr. Bhattacharya.

e-mail: profpkb@yahoo.co.in

Received 12 January 2016

Accepted 3 November 2016 also lead to liver failure and hepatocellular carcinoma (HCC). A study of 53,178 recipients of $\mathrm{HCV}$-contaminated lots of immunoglobulin products in Ireland in 1997 showed that 400 $(0.8 \%)$ were positive in the subsequent 17 years, during the 18 25-year retrospective study period, and liver biopsy revealed mild or moderate hepatitis in $93 \%$, bridging fibrosis in $15 \%$, and cirrhosis in $2 \%$ of the recipients ${ }^{(3)}$. In patients with chronic $\mathrm{HCV}$ infection, death is usually due to cardiac or other diseases; liver-related mortality occurs in approximately $4.1 \%$ of patients with chronic HCV infection, compared with $1.3 \%$ of controls ${ }^{(4)}$.

Liver histology of chronic HCV infection generally consists of intense mononuclear inflammatory infiltrate and some degree of fibrosis from minimal expansion of the portal tract due to cirrhosis. The fibrotic process is driven by liver inflammation and progresses based on environmental, viral load, and host factors. Lymphoid aggregates of follicles with germinal centers are typical of chronic HCV infections. Immunohistology shows that germinal centers contain activated B cells surrounded by a network of follicular dendritic cells and a mantle zone of B cells, which in turn is surrounded by T cells. There are also fatty hepato cellular changes (steatosis) in 50\% of liver biopsies, and patients infected with genotype 3 have more fat due to cytopathic effects by cluster of differentiation 8 (CD8+) T cells. Severe bile duct injury is observed in $10-15 \%$ of biopsies from patients with chronic HCV and HIV-1 coinfection.

Staging of chronic hepatitis requires an assessment of the degree of fibrosis using Masson's Trichrome stain. There are various other systems to grade and stage chronic hepatitis, including the modified Knodell Histology Activity Index (grade, 0-18; stage,0-4) and Ishak score (0-6), which is based on interphase hepatitis, confluent necrosis, parenchymal injury, portal inflammation, and fibrosis. However, the most meaningful assessment of the activity of liver diseases based on the Metavir scoring system, 1994: no fibrosis(F0), portal fibrosis (F1), little bridging fibrosis (F2), considerable bridging fibrosis (F3), and cirrhosis (F4). The inter observer variation of the Metavir system when the pathologist is well trained is low, but the sampling error often exceeds $>30 \%$; the system depends on the length of the liver biopsy obtained by a true cut needle, with at least $1.5 \mathrm{~cm}$ required. 
In an unpublished, retrospective study conducted by this author (as part of a 2003 thesis for MD in Pathology at West Bengal University of Health Sciences), cirrhosis occurred in $16-24 \%$ of patients with post-transfusion HCV infection, over a follow-up of 8-14 years. The author relied on the rate of progression from hepatitis to cirrhosis, based on the fibrosis score (Metavir stage instead of Knodell or Ischak) using the estimated number of years. Using this method, the average rate of fibrosis accumulation to develop cirrhosis was 0.133 units/year. However, the author agrees that many factors may influence the rate of fibrosis and proposes that most patients fall in one of three fibrosis rate groups: rapid $(<20$ years to develop cirrhosis), intermediate (late cirrhosis development), and slow (no cirrhosis even after 50 years). The slow group had a fibrosis rate of 0.5 units/year, which extrapolates to a mean time of 80 years to cirrhosis. Hepatic inflammation also influences the rate of fibrosis; after 20 years, the risk of cirrhosis is $10-13 \%$ with minimal or mild chronic HCV infection and $44-100 \%$ with moderate to severe hepatitis. With bridging fibrosis, the 5 -year risk of cirrhosis becomes 58\%in 10 years and 100\% in 20 years $^{(5)}$. The other factors that accelerate fibrosis and disease progression are regular, heavy alcohol consumption; age ( $>50-55$ years); female gender; hispanic or white ethnicity; human leukocyte antigen DRB; HIV and other immune suppressive conditions; steatosis; severity of inflammation; necrosis and injury; and a high iron load.

HIV-infected patients have higher HCV levels and thus a higher rate of fibrosis progression. Progression to cirrhosis is 3 -fold higher in HIV-infected persons and is more common in patients with low cluster of differentiation 4 (CD4) counts. $\mathrm{HCV}$ viral load does not have a pivotal role, but monitoring disease progression can help to predict treatment response. The $\mathrm{HCV}$ genotype in that locality, as determined using restriction fragment length polymorphism, is important. Improved treatment response is observed with genotypes 2 and 3 than with genotypes 1 and 4, and patients with genotype 1 or 4 require longer treatment periods.

Hepatic fibrosis in hepatitis is a wound-healing response; myofibroblast-like cells produce hepatic fibrosis regardless of the underlying cause, and stellate cells are the key sources of these hepatic myofibroblasts. Stellate cells are actually a heterogeneous population of mesenchymal cells and are the primary site for storing retinoids and key fibrogenic cells in the liver. Stellate cell activation refers to the transformation from quiescent vitamin A-rich cells to highly fibrogenic cells in any type of hepatitis, characterized morphologically by enlargement of rough endoplasmic reticulum, diminution of vitamin A droplets, muffled nuclear membrane, appearance of contractile filaments, and proliferation. Activated stellate cells are seen in large numbers at the region of greatest injury in both HCV and HIV co infection and HCV and hepatitis B confection. Large families of matrix metalloproteinases (MMPs) is responsible for liver fibrosis, of which fibroblast activation proteins such as stromelysin membrane-type metaloproteinases MT1-MMP2, MMP3 TIMP2, and plasmin release fibril-forming type 1, type III, and type Vcollagen. Fibrosis due to chronic liver disease is related with the activity of matrix degrading enzymes, their inhibitors, and fibrogenic stimuli as well relative collagen cross linking. The biologic activity of extracellular matrix is altered, including increased cellular fibronectin, altered integrin behavior (tripeptide sequence Arg-Gly-ASP, i.e.,GRD), cadherin selectin, upregulated tyrosine kinase receptors, discoidin domain receptors (DDR2) for activation of stellate cells that stimulate MMP and platelet-derived growth factor (PDGF), hepatocyte growth factors, connective tissue growth factors(CTGF), tumor necrosis factor- $\alpha$, vascular endothelial growth factor (VEGF), and basic fibroblast growth factor (BFGF).

Now, the question is howstellate cells are activated in $\mathrm{HCV}$ infections. Activation of stellate cells occurs in two phases: initiation and perpetuation. In the initiation phase, gene expression renders stellate cells responsive to cytokines and stimuli, while the perpetuation phase results from stimuli affecting fibrosis generation; initiation largely occurs from paracrine stimulation, and perpetuation occurs from both the autocrine and paracrine loops. Paracrine stimuli originate from neighboring cells such as the sinusoidal endothelium, Kupffer cells, hepatocytes, and platelets (human platelet antigen $1 \mathrm{a} / 1 \mathrm{~b}$ and PDGF), and sinusoidal cells release TGF- $\beta$ in the pro-fibrogenic form when sinusoidal cells release adhesion molecules and VEGF. Kupffer cells stimulate MMPs and release retinoids,reactive oxygen species, and lipid peroxidase, which are strongly fibrogenic in nature. In HCV infection, steatosis occurs and increases stellate cell activation and fibrogenesis through the generation of free radicals.

The phenomenon of perpetuation requires proliferation of stellate cells, chemotaxis, fibrogenesis, contractility, matrix degradation, retinoid loss, and cytokine release; proliferation requires PDGF, VEGF, TGF- $\alpha$, and BFGF signaling pathways. Fibrogenesis requires TGF- $\beta 1$ and CTGF; contractility requires endothelin-1. Retinoid loss requires peroxisome,proliferated activation of receptors[agnathan paired receptors resembling antigen receptors, peroxisome proliferator-activated receptor (PPAR)- $\gamma$ ], farnesoid receptors, and chemoattraction for inflammation of mono and polymorphic cells; for hepatic inflammation, several chemokines such as $\mathrm{CCL}_{2}$, RANTES, $\mathrm{CCR}_{5}, \mathrm{CxCR} 4$, and toll like receptors(TLR3)are important ${ }^{(6)}$. $\mathrm{CD} 8+\mathrm{T}$ cells are more fibrogenic towards stellate cells than $\mathrm{CD}_{4}+\mathrm{T}$ cells, and the HIV $\mathrm{CD}_{4} / \mathrm{CD}_{8}$ ratio is reduced more than with $\mathrm{HCV}$ infection alone. The TLR3 gene plays an important role in the immune response and clinical progression of fibrosis, chronicity, cirrhosis, and HCC in HCV and hepatitis $\mathrm{B}$ viral infections. In chronic viral infections, liver TLR3 expression is increased, leading to hepatic billiard damage and increased GGT levels in viral hepatitis. In HCV infections, putative $\mathrm{HCV}$ receptors are also activated in the stellate cells inducing $\mathrm{CD}_{80}$, $\mathrm{LDL}$ receptors, and $\mathrm{Clq}$. The $\mathrm{E}_{2}$ protein of $\mathrm{HCV}$ interacts directly with $\mathrm{CD}_{81}$ a stellate cell membrane receptor. Chemokines and their receptors are up regulated, stimulating lymphocyte recruitment, statuses, and apoptosis in HCV as well as accelerating fibrosis. There are many transcriptional regulatory proteins of satellite cell activation, of which PAR- $\delta$, $-\beta,-\gamma$, cosine and lysine rich protein 2 (CRP-2), retinoid receptor Nek, Rappel-like factor-6, Foxf1, Lhx, and MEF2 are important ${ }^{(6)}$. 
HIV1 and other hepatitis viruses: TTV and SEN virus are circular, single-stranded deoxyribonucleic acid (DNA) viruses that cause blood borne infections. Nine different SEN virus genotypes (A to I) have been identified, with at least $2.5 \%$ divergence in their nucleotide sequences. The SEN virus has been isolated from the serum of HIV-positive and drug-using patients, and the SEN virus-D and $\mathrm{V}-\mathrm{H}$ genotypes are the most common in patients with HIV1. TTV is also a transfusiontransmitted virus that was first identified in a Japanese patient in 1997; the genome of SEN virus is similar to that of TTV, and both are classified within the circovirus family. According to a number of studies, the prevalence of TTV in HIV patients is slightly higher than in healthy blood donors. The TTV prevalence is higher in Spain, with a prevalence of $76 \%$ among the HIV-infected Spanish population, compared with $61 \%$ in Iran. Among HIV-infected patients, the TTV and SEN virus infection rates are higher than in healthy blood donors, suggesting that blood transfusion is the main transmission route. However, TTV is also detected in the healthy population with no history of blood transfusion, suggesting it can be transmitted in ways other than blood or injections, such as contaminated food, amniotic fluid from TTV-positive women, breast milk, feces, saliva, bites, and a throat swab. The high prevalences of TTV DNA, SEN virus-D (24\%), and SEN virus-H (33\%) are due to contaminated equipment and blood products and result in chronic liver disease (64\%) and liver failure in HIV-infected patients. Therefore, the presence of TTV is an independent risk factor and prognostic factor ${ }^{(7)}$.

\footnotetext{
Acknowledgement

The authors gratefully acknowledge the following persons for their valuable contribution to this article: Upasana Bhattacharya, Rupak Bhattacharya, Ritwick Bhattacharya, Rupsa Bhattacharya, Dalia Mukherjee, Debasis Mukherjee, Oaindrila Mukherjee, and Ayshi Mukherjee (7/51 Purbapalli; PO- Sodepur; Dist 24 Parganas [north] Kolkata-110; West Bengal; India and
}

Runa Mitra of B K Mitra palliative care Barrack pore North 24 Parganas West Bengal; india). All of these contributors agreed to be mentioned in the Acknowledgments section, rather than as authors.

\section{Conflicts of Interest}

The authors declare that there is no conflict of interest.

\section{REFERENCES}

1. Picelli N, Tanikawa AA, Grotto RMT, Silva GF, Barbosa AN, Ferrasi $\mathrm{AC}$, et al. The absence of human platelet antigen polymorphism effect on fibrosis progression in human immunodeficiency virus-1/ hepatitis C virus coinfected patients. Rev Soc Bras Med Trop 2015; 48:406-409.

2. Eystey ME, Diamondstone LS, Lien JM, Ehmann WC, Quan S, Goedert JJ. Natural history of hepatitis C virus infection in multitransfused haemophiliacs: effect of coinfection with human immunodeficiency virus. The Multicenter Hemophilia Cohort Study J Acquir Immune Defic Syndr 1993; 6:602-610.

3. Kenny-Walsh E, The Irish Hepatology Research Group. Clinical outcomes after hepatitis infection from contaminated anti-D immune globulin. N Engl J Med 1999; 340:1228-1233.

4. Seeft LB, Hollinger FB, Alter HJ, Wright EC, Cain CM, Buskell ZJ, et al. Long-term mortality and morbidity of transfusion-associated non A non B and type C hepatitis: A National Heart, Lung, and Blood Institute Collaboration study. Hepatol 2001;33:455-463

5. Yano M, Kumada H, Kage M, Ikeda K, Shimamatsu K, Inoue O, et al. The long-term pathological evolution of chronic hepatitis $\mathrm{C}$. Hepatol 1996; 23:1334-1340.

6. Schiff ER, Sorrel MF, Maddrey-Schiff WC. Hepatic Fibrosis. In:Schiff ER, Sorrel MF, Maddrey WC, editors. Schiff's Diseases of the Liver. $10^{\text {th }}$ edition; vol 1, chapter 14. Philadelphia: Lippincott William \& Wilkins Publisher; 2007. p. 395-418.

7. Pirouzi A, Bahmani M, Feizabadi MM, Afkari R. Molecular characterization of Torque teno virusand SEN virus co infection with HIV in patients from Southern Iran. Rev Soc Bras Med Trop 2014; 47:275-279. 